\title{
Effect of Processing Paramters on Metal Matrix Composites: Stir Casting Process
}

\author{
G. G. Sozhamannan ${ }^{1 *}$, S. Balasivanandha Prabu ${ }^{2}$, V. S. K. Venkatagalapathy ${ }^{1}$ \\ ${ }^{1}$ Department of Mechanical Engineering, Sri Manakula Vinayagar Engineering College, Pondicherry, India; ${ }^{2}$ Department of Me- \\ chanical, Anna University, Chennai, India. \\ Email: *'sozhan30@yahoo.co.in
}

Received September $14^{\text {th }}, 2011$; revised November $1^{\text {st }}, 2011$; accepted November $20^{\text {th }}, 2011$

\begin{abstract}
Conventional stir casting process has been employed for producing discontinuous particle reinforced metal matrix composites for decades. The major problem of this process is to obtain sufficient wetting of particle by liquid metal and to get a homogenous dispersion of the ceramic particles. In the present study, aluminium metal matrix composites were fabricated by different processing temperatures with different holding time to understand the influence of process parameters on the distribution of particle in the matrix and the resultant mechanical properties. The distribution is examined by microstructure analysis, hardness distribution and density distribution.
\end{abstract}

Keywords: Stir Casting; Processing Temperature; Holding Time

\section{Introduction}

Since the early 1960 , there is demand for new and improved engineering materials with advancement of modern technology interest in the areas of aerospace, automotive industries had forced a rapid development of metal matrix composites. High demands on material for better overall performance has led to extensive research and development efforts in the composites fields. Among the composites field, the aluminium based metal matrix composite materials are widely used. To meet emerging need, innovations in materials processing enabled achieving an enhancement in stiffness, realization of high strength to weight ratio, an improvement in wear resistance, maintaining strength at elevated temperatures $[1,2]$.

Aluminium based metal matrix composites have been one of the key research areas in materials processing field in the last few decades. Most of the research work has been dealing with aluminium matrix and $\mathrm{SiC}$ reinforcement requiring the light weight in combination of high strength and high stiffness. This is because aluminium is lighter weight which is first requirement in most of the industries. In additionally, it provides impressive strength improvement and the thermal expansion coefficient of Al matrix composites can be adjusted by using silicon carbide, carbon and boron carbides [3]. As a result, these materials are now being rapidly utilized in industries that traditionally used metals, and these have become the forefront of research and development activ${ }^{*}$ Corresponding author. ity in the many related areas [4].

The foundry casting processes have been a favoured processing method as they lend themselves to the manufacture of large number of complex shaped components. Especially, the stir casting mostly used to produce the PRMMCs because it shown to be a very promising for the manufacture of near net shape composites in a simple and cost effective manner. The major problem in this technology is to obtain sufficient wetting of particle by the liquid metal and to get a homogeneous dispersion of the ceramic particles [5]. Several structural defects such as porosity, particle clustering, oxide inclusions and interfacial reactions were found to arise from the unsatisfactory casting technology [6]. Smeulders et al., (1986) [7] studied the thixotropic behaviour, development of the particle shape, and particle distribution in the metal matrix composites by using stir casting. The particles size distribution depends on the cooling rate and stirring rate. The processing temperature influence the cooling rate and stirring rate. These both parameters influence particles distribution in the matrix. At low stirring rate, diameter of cluster is appeared is smaller than higher stirring rate.

Lloyd (1989) [8] studied the processing parameters such as processing temperatures, holding time, and velocity of $\mathrm{SiCp}$ in liquid melts. This factors influencing the production and microstructures of the cast $\mathrm{Al}-\mathrm{SiCp}$ composite, and also influence the formation reaction products $\left(\mathrm{Al}_{4} \mathrm{C}_{3}\right)$ at the interface of $\mathrm{Al}-\mathrm{SiCp}$, which also 
affects the casting fluidity of composites. Zhou and $\mathrm{Xu}$ (1997) [6] studied the particulate reinforced metal matrix composites by gravity casting and two step methods mixing. They were found the improvement of wettability, particles distribution, and also they found $\mathrm{SiC}$ particles distribution in the interdendritic regions. Rohatgi et al., (1993) [9] made an attempt to study the influence of impeller geometry and baffles on the uniformity of distribution of $\mathrm{SiC}$ in water. This study quantitatively measures the actual $\mathrm{SiC}$ concentration. The objective of the study is the influence of stir casting process parameters such as processing temperature and holding time on the uniform distribution of particles and resulting mechanical properties such as Tensile, ductility, hardness and impact behaviour.

\section{Experimental Work}

The matrix material used for the current study was Al-11Si-Mg alloy, having composition average values provided by the supplier (Sargam metals Ltd., India) in weight percentage as shown in Table 1. The Al-11Si-Mg alloy has an excellent combination of mechanical properties in the cast condition. The matrix material was loaded in a graphite crucible and it was placed inside a top loaded resistance furnace at different temperature level $\left(700^{\circ} \mathrm{C}, 750^{\circ} \mathrm{C}, 800^{\circ} \mathrm{C}, 850^{\circ} \mathrm{C}, 900^{\circ} \mathrm{C}\right)$. The $\mathrm{SiC}$ reinforcement (average size $40 \mu \mathrm{m}$, supplied by universal carborendum Ltd, India) was preheated at $1000^{\circ} \mathrm{C}$ for two hours before added in the matrix melt. The $10 \%$ by volume of preheated $\mathrm{SiC}$ powder was added in the liquid melt and the slurry was consciously stirred using a stirring. The four blade Stirrer was designed in order to produce the adequate homogenous particle distribution throughout the matrix material. Four blades stirrer setup is shown in Figure 1. The axial and radial flows are provided to avoid different stagnant zones in the liquid melt by stirrer. Stirring of the mixture is carried out at different holding time $(10,20$, and 30 minutes) to achieve homogeneity of particulates. The stainless steel stirrer blade was coated with zirconia to avoid the reaction between stainless steel and Al alloys at higher temperatures. The Argon gas was supplied into the near the crucible during the stirring to avoid the formation of oxide layer on the surface of matrix melt. The Stirring speed $450 \mathrm{rpm}$ was maintained throughout work. The mixture is allowed to solidify in the preheated $\left(300^{\circ} \mathrm{C}\right)$ steel die.

Table 1. Composition of aluminium alloy.

\begin{tabular}{cccccccccc}
\hline $\mathrm{Si}$ & $\mathrm{Cu}$ & $\mathrm{Mg}$ & $\mathrm{Ti}$ & $\mathrm{Ni}$ & $\mathrm{Li}$ & $\mathrm{Zn}$ & $\mathrm{Fe}$ & $\mathrm{Mn}$ & $\mathrm{Al}$ \\
\hline 11.12 & 0.68 & 0.73 & 0.009 & 0.002 & 0.027 & 0.002 & 0.31 & 0.32 & remains
\end{tabular}

$\begin{array}{llllllllll}11.12 & 0.68 & 0.73 & 0.009 & 0.002 & 0.027 & 0.002 & 0.31 & 0.32 & \text { remains }\end{array}$

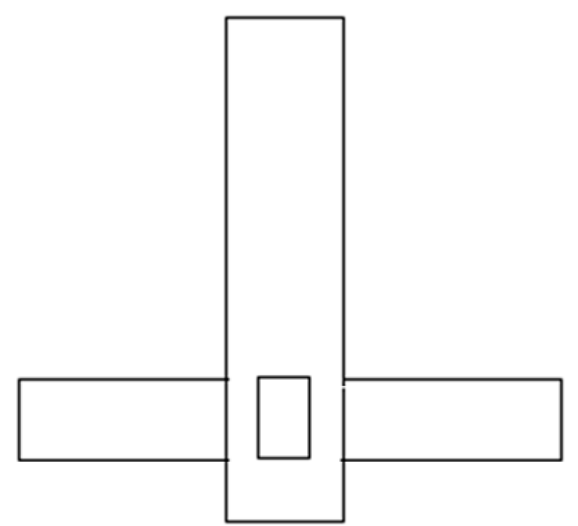

Figure 1. Stirrer blade setup.

\section{Results and Discussion}

\subsection{Structure of $\mathrm{Al}-10 \% \mathrm{SiCp}$ Composites}

The optical micrographs of aluminium composites reinforced with 10\% of SiCp are shown in Figures 2(a)-(e). When the specimen was prepared at $700^{\circ} \mathrm{C}$ and $750^{\circ} \mathrm{C}$, $800^{\circ} \mathrm{C}$, the particles were distributed uniformly and dendrite structure was more obvious in the microsturcture (figure). There is no large pore and particles clustering existed in these SEM micrographs. However, the other two composites (at $850^{\circ} \mathrm{C}$ and $900^{\circ} \mathrm{C}$ ) having pores and particles clustering. The existence of pores and particle clustering was attributed to the high viscosity and low shearing rate of the melt. The viscosity decreased and the shearing rate decreased when increasing process temperatures. Hence, the particles cluster fairly formed in the melt. This can be attributed more particles clustering in the Al matrix.

It can be seen from the figures that there is an increase the particles cluster corresponding to an increase in the processing temperatures. Which were stirred for a constant speed with longer period at higher temperature, the particles were agglomerated in the melt. Although there is an increase in the particle clustering with increase processing temperature, it was observed that the tendency for formation of particle cluster was greater in the higher holding time than in the low holding time. During the higher holding time with temperature, the geometry of the capturing of the particles does not restrict their movement inside the liquid metal as well as solidification. Also the presence of a low viscosity of liquid metal tents to physically not restricts growth of porosity. Thus, the tendency for particle cluster or porosity is high in the higher temperature with prolonged contact between matrix and reinforcement.

\subsection{Effect of Processing Temperatures on Processing Al-SiCp Composites}

The effect of processing temperature is illustrated where 

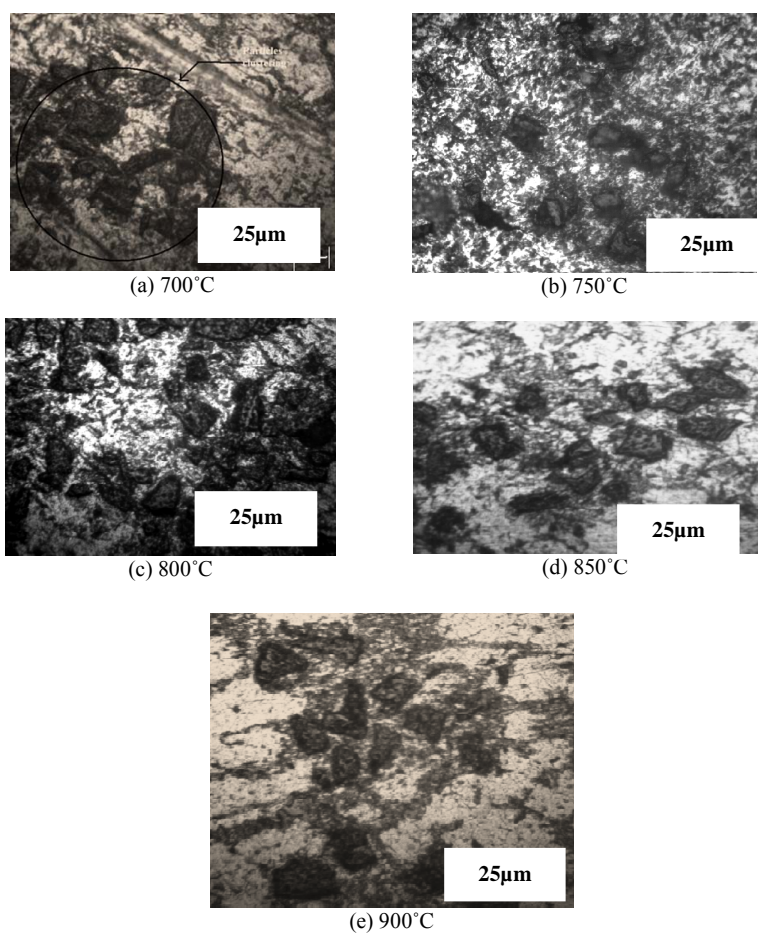

Figure 2. Optical image shows particles distribution at 20 minutes holding time.

the contact time between reinforcement and liquid $\mathrm{Al}$ metal with different holding time. The processing temperature is mainly influencing the change in viscosity of Al matrix and also it accelerating the chemical reactions between them. This change of viscosity was calculated theoretically by using Arrihenious equation [10].

$$
\eta=\eta_{0} \exp \left(\frac{E}{R T}\right)
$$

where $\eta_{0}$-viscosity of aluminium at the melting temperature, E-Activation energy for viscous flow of aluminium, R-Universal gas constant processing temperature. The viscosity of liquid Al matrix with $10 \% \mathrm{SiCp}$ is often calculated with the Einstein function as follows [11]:

$$
\eta / \eta_{0}=1+2.5 C+10.5 C 2+\exp (A B),
$$

in which $A=0.0023, B=16.6, C$ is volume fraction of particles.

The changes in viscosity with respect to various processing temperatures are shown in Figure 3. The change of viscosity is significant, as can be seen by comparing $\mathrm{Al}$ alloy and Al-SiCp composites. The suspending liquid apparent viscosity of $\mathrm{Al}-\mathrm{SiCp}$ is higher by nearly $38 \%$ than the $\mathrm{Al}$ matrix without reinforcement. But, the both viscosity is increased when processing temperature increased from $700^{\circ} \mathrm{C}$ to $900^{\circ} \mathrm{C}$. The changes of viscosity influence the particle distribution in the $\mathrm{Al}$ matrix. At higher viscosity with lower temperature (below $800^{\circ} \mathrm{C}$ ), the geometric contacts of particles is restricted by vortex

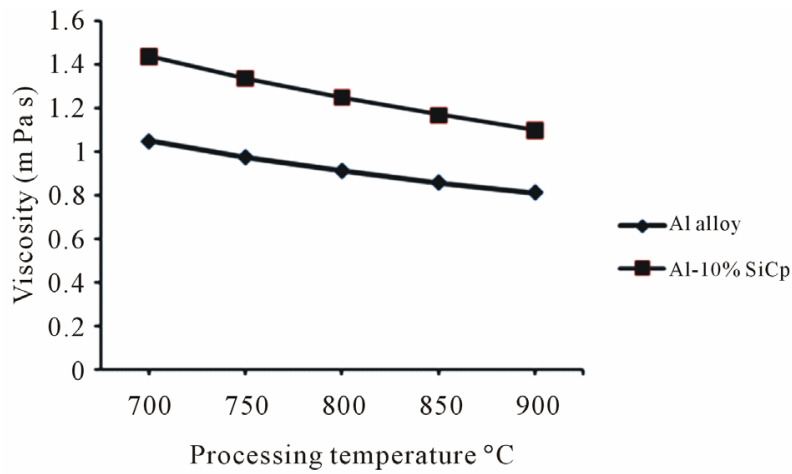

Figure 3. Changes of visosity as function of temeprature.

of molten metal. Therefore, the particles are distributed uniformly in this range. But, at lower viscosity with higher temperature (above $800^{\circ} \mathrm{C}$ ), the geometric of particles are captured and it was unable to restricts by the vortex of molten metal under the constant stirring speed. The conclusion can be drawn that viscosity of liquid decreased when increasing processing temperatures with increasing holding time.

\subsection{Effect of Holding Time}

Effect of the holding time helps in the Al-SiCp composites mainly two ways: to distribute the particles in the liquid, and to create perfect interface bond between reinforcement and matrix. The holding time between matrix and reinforcement is considered as important factor in the processing of composites. When the holding time is 10 minutes, the particles are distributed uniformly in the matrix at $700^{\circ} \mathrm{C}, 750^{\circ} \mathrm{C}$ and $800^{\circ} \mathrm{C}$. The liquid matrix has sufficient viscosity in the temperature range, and velocity of particles flow is small. The similar results are observed in the 20 minutes holding time. In the case of 30 minutes holding time, the liquid has sufficient viscosity at lower temperature $\left(<800^{\circ} \mathrm{C}\right)$ but the contacts time between matrix and reinforcement too large. During this period, the particles are distributed uniformly in liquid even though some of particles form cluster which could be also are located in the matrix region. A vortex created during the stirring can suck the air or gas bubbles in to the liquid metal. As the results, the particles were attached with air bubbles to form the particles cluster in the matrix. At higher temperature $\left(>800^{\circ} \mathrm{C}\right)$, more particles cluster are found in the composite bar.

\section{Effect of Processing Parameters on Mechanical Properties of Al-SiCp}

\subsection{Tensile Properties}

Figure 4 shows the variation in the ultimate tensile strength as a function of temperature is illustrated for Al-10\% SiCp composites with different holding time. 


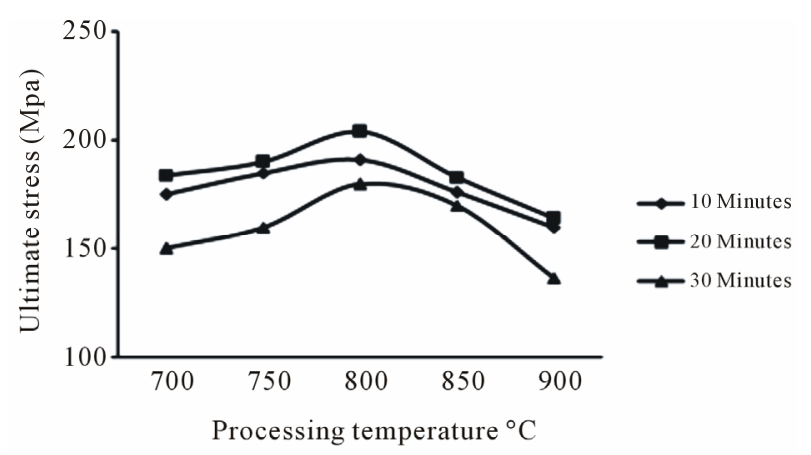

Figure 4. Effect of processing parameters on tensile strength of $\mathrm{Al} / \mathrm{SiCp}$.

The ultimate strength of $\mathrm{Al}-10 \% \mathrm{SiCp}$ composite has been increased and reached a maximum as the processing temperature changes from $700^{\circ} \mathrm{C}$ to $800^{\circ} \mathrm{C}$, then began decrease with further increase of processing temperature from $800^{\circ} \mathrm{C}$ to $900^{\circ} \mathrm{C}$. These composites exhibited different tensile behaviors. The overall strength of composites is influenced by distribution of particles in the matrix.

It is show that the $\mathrm{SiC}$ particles are uniformly distributed in the matrix when the processing temperatures are at $700^{\circ} \mathrm{C}$ to $800^{\circ} \mathrm{C}$ for 10 minutes holding time. It is obvious that the reduction in viscosity on increasing the processing temperatures which is shown in figure during the tensile load, the presences of particles hinder the dislocation movement during plastic deformation and it exhibits the isotropic properties in the matrix. Therefore, the ultimate strength is increased gradually. Further increase in temperature from $800^{\circ} \mathrm{C}$ to $900^{\circ} \mathrm{C}$, the particles cluster associated with porosity is formed in the liquid matrix due to the vortex of molten stirrer which entrapped the gases inside the matrix. The viscosity also decreased further. The clustering of particles has considerable effect on strength and plastic behavior of composites. Elastic response of the composite is not much affected due to clustering nature

\subsection{Hardness Distribution}

The Brinell hardness number was measured along length of the cast specimen at an interval of $1 \mathrm{~cm}$. The low temperature with holding time hardness values at some places is minimum it close to harness values of Aluminium and some places is more. The high values is obtained from the places where the particles is accumulation more and lower hardness values is obtained from places where $\mathrm{SiC}$ particles where absent. The Figure 5 shows the hardness number distributions along length of the cast specimens. The $\mathrm{SiC}$ particles added to the aluminium alloy matrix have a satisfactory effect in improving the hardness of the composites. This is to be expected since

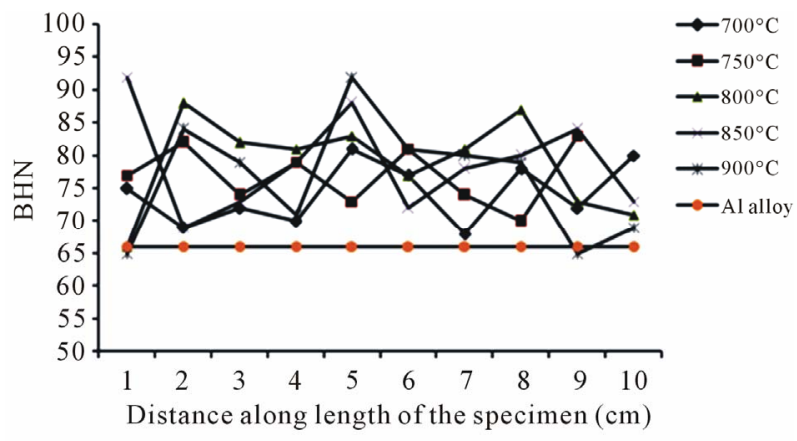

Figure 5. Effect processing temperatures on Hardness of Al/SiCp.

aluminium is a soft material and the SiC particles being hard, contribute positively to the hardness of the composite. The presence of stiffer and stronger particles leads to constrain the plastic deformation of the matrix during the hardness test. But, constrains to the plastic deformation depend on the distribution of the particles in the matrix. Some local regions in the composite show remarkable changes, if the particle accumulated in a particular place the values are higher, and if the particles are absent in some places the values are lower. The hardness measurements were taken in cast specimen to understand the distribution of the $\mathrm{SiC}$ particle in the $\mathrm{Al}$ alloy at different processing conditions.

\subsection{Impact Test}

The results of the charpy impact tests for $\mathrm{Al}-10 \% \mathrm{SiCp}$ composites fabricated with different processing temperatures with holding time are given in Table 2. The test results revealed that the impact energy of $\mathrm{Al}-\mathrm{SiCp}$ is mainly depends on the distribution of the particles in the matrix. It is interesting to note there is a little variation in the value for the different processing conditions as shown in Table 2. The impact values are slightly increases with increasing the processing temperatures. The decrease of impact values at lower temperature in the Al-SiCp composites can be attributed to the presence of brittle $\mathrm{SiCp}$ which may act as stress concentration areas,

Table 2. Impact value of Al-SiCp composites.

\begin{tabular}{|c|c|c|c|}
\hline \multirow{2}{*}{ Processing temperatures $\left({ }^{\circ} \mathrm{C}\right)$} & \multicolumn{3}{|c|}{$\begin{array}{l}\text { Impact values with } \\
\text { different holding time }(\mathrm{J})\end{array}$} \\
\hline & 10 mins. & 20 mins. & 30 mins. \\
\hline 700 & 3 & 4 & 6 \\
\hline 750 & 3 & 6 & 6 \\
\hline 800 & 4 & 6 & 8 \\
\hline 850 & 5 & 8 & 8 \\
\hline 900 & 5 & 10 & 10 \\
\hline
\end{tabular}


where the particles are distributed uniformly.

\section{Conclusions}

The following conclusion can be reached based on the present investigation:

- From the microstructure analysis, the particles were distributed uniformly in the processing temperature $750^{\circ} \mathrm{C}$ and $800^{\circ} \mathrm{C}$. The particles agglomerations were found in the processing temperature of $700^{\circ} \mathrm{C}, 850^{\circ} \mathrm{C}$ $=$ and $900^{\circ} \mathrm{C}$ due to the changes of viscosity in liquid Al matrix.

- The viscosity of Al matrix decreases with increased processing temperatures. The suspending liquid viscosity of Al-SiCp is higher by nearly $38 \%$ than the $\mathrm{Al}$ matrix without reinforcement.

- The tension test revealed that ultimate strength increased gradually up to $800^{\circ} \mathrm{C}$ and starts to decrease gradually due to the distribution in the Al matrix.

- The Ultimate strength of metal matrix composite decreases with increasing holding time. It is revealed that holding time influences the viscosity of liquid metal, particles distribution and also induces some chemical reaction between matrix and reinforcement.

The hardness values increases more or less linearly with increasing of processing temperatures from $750^{\circ} \mathrm{C}$ to $800^{\circ} \mathrm{C}$ at 20 minutes holding time.

\section{REFERENCES}

[1] T. P. Rajan, R. M. Pillai and B. C. Pai, "Review Reinforcement Coatings and Interfaces in Aluminium Metal Matrix Composites," Journal Material Science, Vol. 33, No. 14, 1998, pp. 3491-3503. doi:10.1023/A:1004674822751

[2] M. Jayamathi, S. Seshan, S. V. Kailas, K. Kumar and T. S. Srivatsan, "Influence of Reinforcement on Microstructure and Mechanical Response of a Magnesium Alloy," Current Science, Vol. 87, No. 9, 2004, pp. 1218-1231.
[3] V. K. Lindroos and M. J. Talvitie, "Recent Advances in Metal Matrix Composites," Journal of Material Processing Technology, Vol. 53, 1995, pp. 273-284.

[4] L. M. Tham, M. Gupta and L. Cheng, "Effect of Limited Matrix-Reinforcement Interfacial Reaction on Enhancing the Mechanical Properties of Aluminium-Silicon Carbide Composites," Acta Materialia, Vol. 49, No. 16, 2001, pp. 3243-3253. doi:10.1016/S1359-6454(01)00221-X

[5] J. Hashim, L. Looney and M. S. J. Hashmi, "Metal Matrix Composites: Production by the Stir Casting Method," Journal of Materials Processing Technology, Vol. 119, No. 1-3, 1999, pp. 329-335. doi:10.1016/S0924-0136(01)00919-0

[6] W. Zhou and Z. M. Xu, "Casting of SiC Reinforced Metal Matrix Composites," Journal of Materials Processing Technology, Vol. 63, No. 1-3, 1997, pp. 358-363. doi:10.1016/S0924-0136(96)02647-7

[7] R. J. Smeulders and F. H. Mischgofsky, "Direct Microscopy of Alloy Nucleation, Solidification and Ageing (Coarsening) during Stir Casting," Journal of Crystal Growth, Vol. 76, No. 1, 1986, pp. 151-169. doi:10.1016/0022-0248(86)90021-7

[8] D. J. Lloyd, "The Solidification Microstructure of Particulate Reinforced Aluminium/SiC Composites," Composite Science and Technology, Vol. 35, No. 2, 1989, pp. 159-179.

[9] P. K. Rohatgi, S. Ray, R. A. Sthena and C. S. Narendranath, "Interface in Cast Metal Matrix Composites," Materials Science and Engineering, Vol. 162, No. 1-2, 1993, pp. 163-174. doi:10.1016/0921-5093(90)90041-Z

[10] F. Miani and P. Matteazzi, "Estimation of Viscosity in under Cooled Liquid Metal Alloys," Journal of Noncrystalline Solids, Vol. 143, 1992, pp. 140-146. doi:10.1016/S0022-3093(05)80561-7

[11] J. Wang, Q. X. Guo, M. Nishio, H. Ohawa, D. Shu and K. $\mathrm{Li}$, "The Apparent Viscosity of Fine Particle Reinforced Composite Melt," Journal of Materials Processing Technology, Vol. 136, No. 1, 2003, pp. 60-63. doi:10.1016/S0924-0136(02)00919-6 